$(\mathrm{BNP}>80 \mathrm{pg} / \mathrm{ml})$ and $\mathrm{B}$ group $(\mathrm{BNP}<80 \mathrm{pg} / \mathrm{ml})$. The incidence of cardiovascular events during hospitalisation and at 6 months after PCI was analysed.

Results Cardiovascular events during hospitalisation in A group included 12 cases, of which 3 died, heart failure occurred in 7 cases; recurrence myocardial infarction and angina in 1 case. 6 months after PCI, there were 4 cases of patients with heart failure and angina occurred in 5 cases. In group B, there were 7 cases of cardiovascular events, 0 deaths, 5 cases of heart failure, angina occurred in 2 cases. Six months later, cardiovascular events occurred in 6 cases, non-cardiac death in 2 cases; heart failure in 3 cases and angina in one. Compared with B group, the incidence of cardiovascular events in A group was significantly higher during hospitalisation $(\mathrm{p}<0.01)$, but no different at 6 months after PCI ( $>>0.05)$

Conclusion BNP concentration in patients with ACS at $48 \mathrm{~h}$ after PCI can only predict short-term prognosis, but cannot predict the long-term prognosis.

\section{E0383 BASELINE CHARACTERISTICS OF MYOCARDIAL INFARCTION SECONDARY PREVENTION STUDY IN TRADITIONAL CHINESE MEDICINE (MISPS-TCM)}

\section{doi:10.1136/hrt.2010.208967.383}

Xu Hongjuan, Ren Ming, Zhang Junhua, Cao Hongbo, Wang Hui, Zheng Wenke, Zhang Boli. Tianjin University of Tcm, Tianjin, China

Objective To evaluate Qishenyiqi Dripping Pills (OSYO) on the efficacy of secondary prevention of myocardial infarction, improvement the evaluation system of coronary heart disease in traditional Chinese medicine (TCM), and to further establish the research methods and corresponding technical specifications of evidence-based medicine in TCM.

Method MISPS-TCM, (a multicenter, large sample, central randomised system, double-blinded, double dummy and positive drug) controlled parallel trials conducted at 18 centers and 88 hospitals in China, which enrolled patients aged 75 years or younger with a history of AMI between 28 days to 2 years, and complicated with syndromes of Qi-deficiency and blood-stasis. The sample size estimated to be 3600 patients were randomly divided into two groups according to the ratio of 1:1, the treatment group taking QSYQ and aspirin simulation, and the control group taking aspirin and OSYO simulation for 18 months. The primary endpoint was non-fatal reinfarction or stroke, or cardiac death, combined with angina pectoris and TCM symptom scores and the Seattle Angina Questionnaire.

Results From November 20, 2005 to October 16, 2008, 3505 patients with AMI were entered into the study: 1746 were assigned to OSYQ, and 1759 were assigned to aspirin. Final follow-up visits were conducted on April 20, 2010. The study had 2451 men (69.9\%). The mean age was 58.3 (SD: 9.0). The mean duration was 9.5 months (SD: 7.2). Disease history: 44\% patients had hypertension, 39\% had hyperlipidaemia, 13\% had diabetes, 12\% had gastritis and so on. Drug treatment history: $80 \%$ were taking antiplatelet drugs, $72 \%$ taking nitrates, with $\beta$ receptor blocking $45 \%$, cholesterol lowering agents present in $41 \%$, ACEI/ARB 38\% and so on. The male ratio, height, smoking and drinking in OSYQ group were slightly lower than in aspirin, however, the other baseline characteristics bore no difference between the two groups, such as demographic information, diagnosis history, medication history, risk factors, angina and TCM symptom scores and the Seattle Angina Questionnaire.

Conclusion Baseline characteristics were well balanced between the two groups that for further analysis and evaluation.

\section{e0384 THE ANALYSIS OF CLINICAL AND CORONARY ANGIOGRAPHIC CHARACTERISTICS FOR PRE-MENOPAUSAL WOMEN WITH CORONARY HEART DISEASE}

doi:10.1136/hrt.2010.208967.384

Ting Wang, Peiling Cong, Xiaofei Sun. Affiliated First People's Hospital of Jining of Shandong, Academy of Medical Sciences, China

Objective To analyse the clinical and coronary angiographic characteristics for pre-menopausal women with coronary heart disease in Jining City, Shandong Province.

Methods 85 female patients with pre-menopausal coronary angiography (CAG) were chosen in Jining First People's Hospital from May 1, 2001 to May 30, 2010. The patients were divided into Coronary Heart Disease (CHD)group and non-CHD group according to coronary angiography. According to $\geq 50 \%$ stenosis of coronary artery, the patients were divided into three groups: single vessel disease, double vessel disease and triple vessel disease. Risk factors for $\mathrm{CHD}$ and angiographic characteristics were analysed. WHO diagnostic criteria was used in the diagnosis of hypertension and diabetes.

Result 31 patients were diagnosed with coronary heart disease, 54 patients diagnosed with normal coronary artery. Comparison of risk factors between the two groups: patients with hypertension were $49.4 \%$ vs $31.0 \%$, with diabetes were $14.3 \%$ vs $3.1 \%$, hyperlipidaemia was $53.6 \%$ vs $25.1 \%$, coronary heart disease risk factor's family history $37.8 \%$ vs $12.6 \%$. Results were above target and there was a significant difference between the groups. The family history of coronary heart disease $27 \%$ vs $19.1 \%$ showed no difference between the two groups. Typical angina symptoms more common in CHD group, mainly those with acute coronary syndrome. Single-vessel disease, coronary artery disease are more common, with an occurrence rate of $70.97 \%$ (22/31), two vessel disease $19.35 \%(6 / 31)$, three vessel disease $0.97 \%$ (3/31). Anterior descending artery involvement is most likely occurring at a rate of $96.77 \%$ (30/31), lesion calcification was much lower in rate of occurrence due to the narrowing of the main limitations.

Conclusion Hypertension, diabetes, hyperlipidaemia and family history of coronary heart disease are risk factors. These risk factors are not as significant a risk factor in pre-menopausal women with coronary heart disease. Coronary artery disease occurs mainly in single-vessel diseased. With the appearance of typical clinical symptoms of angina and multiple risk factors, especially a family history of pre-menopausal female patients, the possibility of major coronary heart disease is larger. For a clear diagnosis, CAG should be performed as soon as possible, in order to avoid misdiagnosis and missed diagnosis. For some patients Target lesion revascularization is necessary.

\section{e0385 ANKLEBRACHIAL INDEX AS A PREDICTOR FOR THE SEVERITY OF CORONARY ARTERY STENOSIS}

doi:10.1136/hrt.2010.208967.385

${ }^{1}$ Chang Xuewei, ${ }^{1}$ Pan Guangjie, ${ }^{1} \mathrm{Gu}$ Yunfei, ${ }^{1} \mathrm{Ma}$ Huifang, ${ }^{1}$ Tian Liping, ${ }^{1}$ Zhang Shouyan, ${ }^{2}$ Wei Yidong, ${ }^{3}$ Wei Jinghan. ${ }^{1}$ Department of Cardiology, Luoyang Central Hospital Luoyang; '2Department of Cardiology, Shanghai Tenth People's Hospital Affiliated to Tongji University, China; ${ }^{3}$ Department of Cardiology, The First Affiliated Hospital of Zhengzhou University

Objective To study the relationship between ankle-brachial index $(\mathrm{ABI})$ and the severity of coronary artery stenosis.

Method This study enrolled 180 patients, who underwent coronary angiography and ABI measurement in addition to date collection regarding cardiovascular risk factors. They were divided into two 
groups according to ABI. The correlation between cardiovascular risk factors, $\mathrm{ABI}$ and the $\mathrm{CAD}$ severity were analysed.

Results Gensini score $(69.0 \pm 20.1)$, three vessel $(56,56 \%)$ and $B_{2} / C$ type stenotic lesion $(79,79 \%)$ was significantly higher in $\mathrm{ABI}<0.9$ group compared with control group (50.7 \pm 17.6$)$, (19, 23.8\%), (31, $38.8 \%)$.

Result Gensini score (69.0 \pm 20.1$)$, threevessel (56, 56\%) and $\mathrm{B}_{2} / \mathrm{C}$ type stenotic lesion $(79,79 \%$ ) was significantly higher in $\mathrm{ABI}<0.9$ group, Compared with control group (50.7 \pm 17.6$)$, (19, 23.8\%), (31, 38.8\%). Binary regression analysis showed that $\mathrm{ABI}$, correlated with several risk factors, was a statistically significant independent predictor for three vessel or complex $\left(B_{2} / C\right)$ stenotic lesions and odds ratio were 3.620 and 4.011 respectively. The stepwise multivariable regression analysis shown, $A B I<0.9(R=-3.018, P<0.05)$ and age $(R=1.206$, $\mathrm{P}<0.05)$ enter the gensini score regression equation.

Conclusion $\mathrm{ABI}$ is negatively correlated with the degree of coronary artery stenosis. $\mathrm{ABI} \leq 0.9$ is a good predictor of severe coronary artery disease.

\section{E0386 EVALUATION OF CORONARY FLOW VELOCITY RESERVE IN HOMOZYGOUS FAMILIAL HYPERCHOLESTEROLAEMIA BY TRANSTHORACIC DOPPLER ECHOCARDIOGRAPHY AND DUALSOURCE CT}

doi:10.1136/hrt.2010.208967.386

Yang Ya, Wang Zheng, Zhang Xiaoshan, Li Rongjuan, Ren Hongyan, Li Zhian. Department of Ultrasound Beijing Anzhen Hospital Capital Medical University

Objective Homozygous familial hypercholesterolaemia (HoFH) is a rare disorder characterised by the early onset of atherosclerosis and usually occurrs at the ostia of coronary arteries. In this study we used transthoracic Doppler echocardiography (TTDE) to evaluate the dynamic changes of coronary flow in $\mathrm{HoFH}$ patients and to detect aortic and coronary atherosclerosis by dual-source CT (DSCT).

Method $20 \mathrm{HoFH}$ patients (12 females, 8 males, mean age $13.1 \pm 5.3$ years, with a mean low density lipoprotein (LDL) cholesterol $583 \pm 113 \mathrm{mg} / \mathrm{dl}$ ) was studied as experimental group and fifteen patients ( 8 females, 7 males, mean age $15.2 \pm 6.9$ years, with a mean low density lipoprotein (LDL) cholesterol $128 \pm 71 \mathrm{mg} / \mathrm{dl}$ ) as control group by TTDE and DSCT.

Result None of the patients showed evidence of ischaemia with standard exercise testing. Though the baseline coronary flow was similar between HoFH patients and normal controls, the hyperaemic flow velocities and thus the coronary flow velocity reserve (CFVR) were significantly lower in those with HoFH (3.36 vs 1.92 respectively, $\mathrm{p}<0.0001)$. All $\mathrm{HoFH}$ patients had aortic plaques, amongst which nine of them occurred at the coronary artery ostia, who had significantly higher LDL-cholesterol and lower CFVR than those without ostia plaques.

Conclusion Our data demonstrated that TTDE together with DSCT could be a useful non-invasive method for detection of coronary flow dynamics and atherosclerosis specifically in HoFH subjects with coronary ostia involved.

\section{e0387 PERCUTANEOUSLY INTERVENTION WAS SUCCESSFULLY PERFORMED IN RIGHT CORONARY ARTERY COMBINED WITH RIGHT SINUS OF VALSALVA ANEURYSM}

doi:10.1136/hrt.2010.208967.387

${ }^{1}$ Liu Fan, ${ }^{1}$ Lu Jingchao, ${ }^{2} \mathrm{Cui}$ Wei, ${ }^{2} \mathrm{Gu}$ Guogiang, ${ }^{2}$ Yang Xiuchun, ${ }^{2} \mathrm{Xie}$ Ruiqin, ${ }^{2} \mathrm{Hao} \mathrm{Jie}$ ${ }^{2}$ Pei Weina, ${ }^{2} \mathrm{Li}$ Baohua. ${ }^{1}$ The Second Hospital of Hebei Medical University; ${ }^{2}$ The Second Hospital of Hebei Medical University

Introduction Sinus of Valsalva aneurysms (SVA) are rare cardiac anomalies. They may be congenital or acquired and are usually asymptomatic unless complicated by such developments as intracardiac rupture with formation of a fistula or aortic valvular insufficiency. The following case is unusual in that the presenting symptom was angina pectoris due to severe atherosclerotic coronary disease with development of an aneurysm of the sinus of Valsalva secondary to atherosclerotic involvement of the aorta.

Case report A 50-year-old male patient presented with 2-week history of increasing exertional chest pain and intermittent chest pain at rest for 1 week. Echocardiography as well as contrast enhanced aorta CT revealed dilatation and irregular protrusion of the right sinus of Valsalva encroaching on right ventricular inflow tract. The aneurysm of the right sinus of Valsalva was about $4.2 \mathrm{~cm}$ in diameter. The aortic valve annulus and aortic valve leaflets were normal. Besides that, contrast enhanced aorta CT showed multiple spotted or comma calcification at aneurysm wall, and at the whole range of aorta accompany by irregular intima thickness. In addition, Selective coronary angiography showed 95\% discrete diameter stenosis at the proximal segment of right coronary artery. A $50 \%$ and a $70 \%$ tubular diameter stenosis were separately noted at the proximal segment of left descending artery and at the origin of first diagonal branch. With these findings, severe coronary atherosclerosis combined with unruptured aneurysm of the right sinus of valsalva was diagnosed. Percutaneously coronary intervention (PCI) was successfully performed, and one drug-eluted stent was deployed in the proximal right coronary artery. There was no dissection at the localisation of stent and aortic root.

Conclusions In general, the surgical method for treating severe coronary heart disease associated with unruptured SVA generally consists of coronary artery bypass grafting and closure of the opening of the aneurysm. To our knowledge, it is the first report that percutaneously intervention was successfully performed in right coronary artery combined with right sinus of valsalva aneurysm. Thereby, PCI may be an alternative treatment strategy for this clinical situation.

\section{e0388 EFFECT OF ENDOTHELIAL PROGENITOR CELLS FROM PERIPHERAL BLOOD IN PATIENTS WITH CORONARY HEART DISEASE BEFORE AND AFTER EXTRACORPOREAL CARDIAC SHOCK WAVE THERAPY}

doi:10.1136/hrt.2010.208967.388

H Y Cai, Y Wang, L Li, L Zhao, X Y Yang, J M Xiao, J M Cao, X Y Yang, C H Yang, T Guo. Department of Cardiology, First Affiliated Hospital of Kunming Medical Colledge, Kunming, China

Objective To study the effects of CSWT on the proliferation, differentiation of endothelial progenitor cells (EPCs) from peripheral blood in patients with coronary heart disease (CAD).

Methods Total mononuclear cells were isolatedfrom patients with coronary heart disease $(n=25)$ and age-matched control subjects $(n=25)$ by Ficoll density gradient centrifugation, and then the cells were plated on fibronectin coated culture dishes. After 7 days of culture. EPCs were characterised as adherent cells double positive for DiLDL-uptake and lectin binding by direct fluorescent staining under alaser scanning confocal microscope.

Results The number and proliferation of EPCs was significantly increased in patients CSWT as compared with that in control subjects $(p<0.05)$, CSWT could markedly promote the proliferation and the differentiation towards the endothelial cell lineage of EPCs in $\mathrm{CAD}$

Conclusion CSWT could markedly promote the proliferation and the differentiation towards the endothelial cell lineage of EPCs in $\mathrm{CAD}$. 